\title{
Um Quiz Game para a Revisão de Conhecimentos em Gerenciamento de Projetos
}

\author{
Giani Petri, Paulo E. Battistella, Christiane G. von Wangenheim, Fernando T. \\ Cassettari, Jean C. R. Hauck \\ Grupo de Qualidade de Software (GQS) - Departamento de Informática e Estatística \\ (INE) - Universidade Federal de Santa Catarina (UFSC) \\ Florianópolis - SC - Brasil \\ giani.petrieprosgrad.ufsc.br, paulo@incod.ufsc.br, \\ c.wangenheim@ufsc.br, fertaranto@gmail.com, jean.hauck@ufsc.br
}

\begin{abstract}
The current scenario of the software sector has need for qualified professionals, including project managers. In this respect, educational games have become an innovative instructional strategy for computing education. Thus, the objective of this paper is to develop an educational quiz game to review the knowledge on project management. The game is developed following the ENgAGED games development process and evaluated by case studies using the MEEGA model. Evaluation results indicate the potential of the game in review the project management knowledge, attracting the interest of students and keeping them motivated and immersed in the learning activity.
\end{abstract}

Resumo. O cenário atual do setor de software está necessitando de profissionais qualificados, incluindo gerentes de projetos. Diante disto, jogos educacionais se tornaram uma estratégia de ensino inovadora para o ensino de computação. Assim, o objetivo deste trabalho é desenvolver um jogo educacional do tipo quiz game para revisar os conhecimentos sobre gerenciamento de projetos. O jogo é desenvolvido seguindo o processo de desenvolvimento de jogos educacionais ENgAGED e avaliado por meio de estudos de caso utilizando o modelo MEEGA. Os resultados das avaliações evidenciam o potencial do jogo em revisar os conhecimentos de gerenciamento de projetos, despertando o interesse dos alunos e mantendo-os motivados e imersos na atividade de aprendizagem.

\section{Introdução}

O crescente cenário do mercado brasileiro de software [ABES, 2015] evidencia a necessidade de qualificação dos profissionais da área, inclusive de gerentes de projetos. Atualmente, a maioria dos projetos de software apresentam problemas [Standish Group, 2015]. Aproximadamente $52 \%$ dos projetos precisam de alteração, $19 \%$ falham por completo e, apenas, $29 \%$ são completados de forma satisfatória, respeitando o prazo e orçamento planejado [Standish Group, 2015]. Este cenário demonstra que há uma deficiência no gerenciamento de projetos de software.

No ensino superior, em cursos da área de Computação, a gerência de projetos é ensinada em disciplinas específicas ou como um tópico menor em disciplinas de Engenharia de Software [ACM/AIS/IEEE-CS, 2005]. Tipicamente a gerência de 
V Congresso Brasileiro de Informática na Educação (CBIE 2016)

Anais do XXVII Simpósio Brasileiro de Informática na Educação (SBIE 2016)

projetos é ensinada por meio de aulas expositivas, que são adequadas para apresentar informações e conceitos. Mas, por ser uma estratégia centrada no professor, não apoia a aplicação prática de competências [Parsons, 2011]. No entanto, considerando o perfil dos estudantes de computação atualmente [Parsons, 2011], é necessário utilizar estratégias de ensino focadas no aluno, permitindo que eles aprendam fazendo, por meio de suas próprias experiências.

Neste contexto, os jogos educacionais tornaram-se uma estratégia inovadora para o ensino de computação [Backlund and Hendrix, 2013; Connolly et al., 2012]. Jogos educacionais são projetados especificamente para ensinar as pessoas sobre um determinado assunto, expandir e revisar conceitos, ou ajudá-las a aprender uma habilidade ou mudança de atitude [Dempsey et al., 1996]. Há vários jogos educacionais em diversas áreas da computação, tais como Programação [Silva et al., 2014, Silva and Dantas, 2014], Estrutura de Dados [Battistella et al., 2012], Engenharia de Software [Ferreira et al., 2014, Medeiros et al., 2013] e Gerenciamento de Projetos [Gresse von Wangenheim et al., 2013]. No entanto, jogos educacionais do tipo perguntas e respostas (quiz games) ainda são pouco utilizados, e os existentes não abordam o conteúdo de gerenciamento de projetos [Wang et al., 2008; Natvig and Line, 2004]. A justificativa para o uso de quiz games é que eles possibilitam um feedback imediato na revisão de conceitos abordados nas perguntas do quiz, permitem uma fixação do conteúdo aprendido e ainda, podem trazer um elemento motivador quando utilizados juntamente com aulas expositivas [Germano et al., 2011].

Diante disto, este trabalho objetiva desenvolver um jogo educacional do tipo quiz game para revisar os conhecimentos sobre gerenciamento de projetos. O jogo é desenvolvido seguindo as etapas do processo de desenvolvimento de jogos educacionais ENgAGED [Battistella and Gresse von Wangenheim, 2015a] e avaliado por meio do modelo de avaliação de jogos educacionais MEEGA [Savi et al., 2011].

\section{Metodologia}

A metodologia de pesquisa adotada neste trabalho divide-se em três etapas principais: levantamento do estado da arte; desenvolvimento do jogo e; avaliação do jogo.

A primeira etapa objetiva identificar o estado da arte dos jogos educacionais digitais do tipo quiz game para ensino de gerenciamento de projetos. Para isso, é realizada uma Revisão Sistemática da Literatura (RSL) segundo Kitchenham (2010). A segunda etapa aborda o desenvolvimento do quiz game. O jogo é desenvolvido seguindo o processo de desenvolvimento de jogos educacionais ENgAGED [Battistella and Gresse von Wangenheim, 2015a], que inclui as fases de: análise, projeto, desenvolvimento, execução e avalição. Na terceira etapa da pesquisa um estudo de caso é realizado com objetivo de avaliar o jogo nos seguintes aspectos: motivação, experiência do usuário e aprendizagem. Para a avaliação foi utilizado o modelo de avaliação de jogos educacionais MEEGA [Savi et al., 2011].

\section{Trabalhos Relacionados}

Como resultado do levantamento do estado da arte, realizado por meio de uma RSL [Kitchenham, 2010], foram encontrados dois quiz games educacionais: Lecture Quiz [Wang et al., 2008] e Age of Computers [Natvig and Line, 2004]. O jogo LectureQuiz 
[Wang et al., 2008] possui os objetivos de aprendizagem focados na revisão dos principais tópicos de arquitetura de software. Ao iniciar o jogo o computador do professor, utilizando um datashow, apresenta uma pergunta e uma série de respostas. Assim que estas respostas aparecerem, o jogador pode escolher a resposta correta a partir de seu dispositivo (computador ou celular). O jogo foi projetado com foco no ensino superior, para alunos da disciplina de Arquitetura de Software, com modo de interação multiplayer. O jogo está disponível somente no idioma inglês e tem duração de 5 a 20 minutos. Em termos de avaliação, foram realizados testes de usabilidade e utilidade com os jogadores, reportando resultados satisfatórios.

No jogo Age of Computers [Natvig and Line, 2004], o jogador vivencia a história do computador. O jogador deve solucionar diferentes tipos de problemas para ganhar pontos. Os objetivos de aprendizagem do jogo estão focados no ensino de fundamentos computacionais. $\mathrm{O}$ feedback ao jogador é realizado no acréscimo da sua pontuação, caso acerte as perguntas. O jogo foi projetado para alunos de cursos da área de computação, é multiplayer e está disponível somente no idioma Norueguês. Em termos de avaliação, foi realizado um estudo com estudantes do Instituto Norueguês de Tecnologia e Ciência, na disciplina de Fundamentos da Computação. Os alunos opinaram em relação ao efeito educativo com mais de $60 \%$ de feedback positivo.

O resultado do levantamento do estado da arte mostrou que na área de gerenciamento de projetos não foram encontradas instâncias de quiz games. Deste modo, optou-se pelo desenvolvimento de um quiz game educacional para a revisão de conhecimentos de gerenciamento de projetos. A descrição completa dos resultados da RSL e o protocolo de busca é detalhado em [Cassettari, 2015].

\section{Concepção do Jogo Kahoot! PMQuiz}

Esta seção apresenta o desenvolvimento do jogo Kahoot! PMQuiz, de acordo com o processo de desenvolvimento de jogos educacionais ENgAGED.

\subsection{Análise}

Análise de aprendizes e contexto. A definição do perfil dos aprendizes e o contexto de aplicação foi realizada com base nos resultados de um survey realizado pelos autores [Battistella and Gresse von Wangenheim, 2016]. O objetivo do survey é identificar o perfil dos jogadores de jogos na área de computação. Com base nos resultados do survey identificou-se que a faixa etária dos aprendizes está em sua maioria (75\%) entre 19 e 30 anos. O público é predominantemente masculino (85\%) e tipicamente preferem jogos de computadores ou consoles (80\%), em comparação com jogos mobile (13\%) e nãodigitais (5\%). Analisando o contexto de aplicação do jogo, o mesmo será aplicado em um laboratório de informática da universidade. Em termos de recursos do laboratório, a sala possui datashow e entre 10 a 20 computadores. Em termos de recursos providos pelos alunos, a maioria (90\%) leva seus dispositivos móveis para sala de aula todos os dias. Destes, $36 \%$ conseguem conectar na internet da universidade sempre, outros $57 \%$ relataram conseguir somente às vezes.

Objetivos de aprendizagem. Após a aplicação do jogo Kahoot! PMQuiz na unidade instrucional, os alunos serão capazes de: revisar os conceitos sobre uma das áreas de 
V Congresso Brasileiro de Informática na Educação (CBIE 2016)

Anais do XXVII Simpósio Brasileiro de Informática na Educação (SBIE 2016)

conhecimento de gerenciamento de projetos (tempo, custo, escopo, riscos, partes interessadas, qualidade, recursos humanos, integração, comunicações ou aquisições) ou um dos grupos de processos de gerenciamento de projetos (iniciação, planejamento, execução, monitoramento ou encerramento).

As perguntas sobre o grupo de processo ou área de conhecimento a ser abordado no quiz game podem ser customizadas pelo professor. No entanto, para este trabalho, o quiz game está focado na revisão de conceitos sobre a área de conhecimento de escopo.

\subsection{Projeto}

Este quiz game será aplicado na unidade instrucional assim que os conteúdos tenham sido introduzidos aos alunos. No caso das disciplinas INE5427 (Planejamento e Gestão de Projetos) e INE5617 (Gerência de Projetos), o jogo será aplicado após o conteúdo da área de conhecimento de escopo de gerenciamento de projetos tenha sido exposto.

Através do uso do laboratório, notebooks ou smartphones pessoais, o jogo pode ser aplicado e os alunos poderão revisar o conteúdo, respondendo às perguntas do quiz. Os alunos poderão obter um feedback sobre o conhecimento dos conteúdos, e então consultar o professor antes da avaliação formal caso haja dúvidas, assim como o professor pode notar alguma dificuldade dos alunos, e então conseguir trabalhar com estas dificuldades.

\subsection{Desenvolvimento}

Esta fase tem por objetivo descrever como ocorreu o desenvolvimento do jogo educacional. Assim, são definidos: os objetivos e características do jogo, a game engine utilizada e o funcionamento do jogo. A Tabela 1 apresenta as características gerais do Kahoot! PMQuiz.

Tabela 1. Características do Kahoot! PMQuiz

\begin{tabular}{|l|l|}
\hline Objetivo do jogo & $\begin{array}{l}\text { O jogador deve responder cada questão dentro de um tempo limite. O jogador que fizer mais pontos } \\
\text { respondendo mais perguntas corretas no menor tempo é o vencedor. }\end{array}$ \\
\hline Gênero & $\begin{array}{l}\text { Quiz game: Um jogo onde o jogador precisa responder perguntas para uma determinada área de } \\
\text { conhecimento. }\end{array}$ \\
\hline Plataforma & Jogo digital de computador online, usando computador/smartphone pessoal com acesso à internet. \\
\hline $\begin{array}{l}\text { Modo de } \\
\text { interação }\end{array}$ & Multiplayer competitivo: vários jogadores competem entre si. \\
\hline
\end{tabular}

Game engine. Durante a revisão da literatura, foram identificados quiz games educacionais desenvolvidos e disponibilizados em plataformas que permitem a alteração das perguntas e respostas, possibilitando aos professores criar seu próprio conjunto de perguntas para aplicar na sua unidade instrucional. Deste modo, optou-se por utilizar uma destas ferramentas para customizá-la e então desenvolver um quiz game para auxiliar na revisão de conhecimentos sobre gerenciamento de projetos. A plataforma escolhida para ser usada neste trabalho foi o Kahoot (https://getkahoot.com/). O Kahoot se destacou e foi escolhido por sua facilidade de manutenção, layout agradável, boa usabilidade, possibilidade de uso online, adaptabilidade para dispositivos móveis, presença dos relatórios dos quiz aplicados e interatividade. O Kahoot é uma plataforma de quiz games web, onde é possível cadastrar grupos de perguntas e respostas, lançar sessões de jogos para os jogadores jogarem e convidar jogadores para estas sessões, onde o jogador poderá responder às perguntas do quiz game. 
V Congresso Brasileiro de Informática na Educação (CBIE 2016)

Anais do XXVII Simpósio Brasileiro de Informática na Educação (SBIE 2016)

A customização da plataforma para o desenvolvimento do jogo incluiu a inserção de perguntas retiradas de exames de certificação PMP (Project Management Professional) [PMI, 2016] e também utilizadas em provas anteriores aplicadas nas disciplinas. Adicionalmente, algumas configurações precisaram ser realizadas na plataforma, como o padrão de respostas (com 4 opções), limite de tempo para cada pergunta, entre outras.

Funcionamento do jogo. Para a execução do jogo, ao selecionar o Kahoot! PMQuiz na plataforma, o professor receberá um código (game-pin). Este código deverá ser passado para os jogadores/alunos que, ao acessarem o link do jogo conseguirão acesso a este jogo específico. Sugere-se que esta tela seja projetada no datashow da sala, para visualização de todos alunos (vide Figura 1). A inserção do game-pin não requer login dos jogadores, somente a inserção de um nome (nick) que valerá somente para esta sessão do jogo. Cabe ressaltar que os alunos podem utilizar o navegador de seu smartphone ou do computador do laboratório de informática. Enquanto os alunos inserem seu nome (nick) a tela do professor (projetada no datashow) automaticamente irá mostrando os alunos já logados no jogo. A Figura 1 apresenta o jogo esperando para ser iniciado, mostrando o game-pin e a lista de alunos já logados.

Após a confirmação do professor (por meio do botão start now), há o início do jogo. Para cada pergunta, a tela projetada pelo professor irá apresentar a pergunta durante o tempo configurado e nos dispositivos dos alunos irão aparecer as quatro cores, referentes as quatro opções de resposta, onde o aluno deverá selecionar uma resposta apenas. Ao final de cada pergunta, aparecerá na tela do professor (projetada no datashow) a resposta correta e a quantidade de alunos que responderam a cada alternativa. Em seguida, será mostrado também o ranking de pontos dos jogadores até o momento. A Figura 2 mostra uma pergunta do jogo Kahoot! PMQuiz, a resposta correta e a quantidade de acertos.

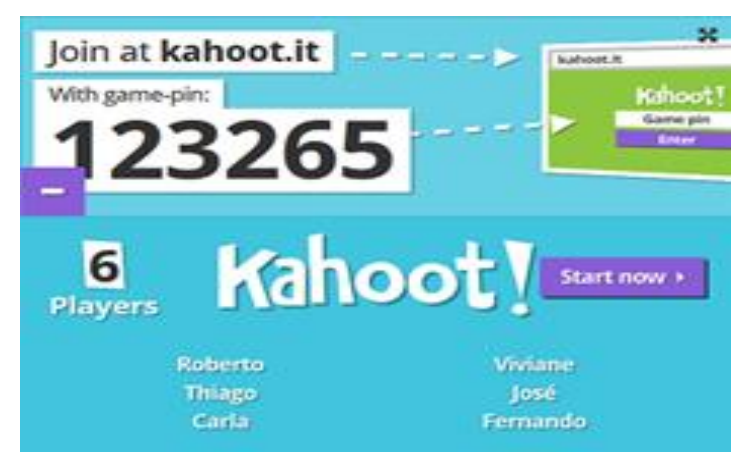

Figura 1. Jogo em espera

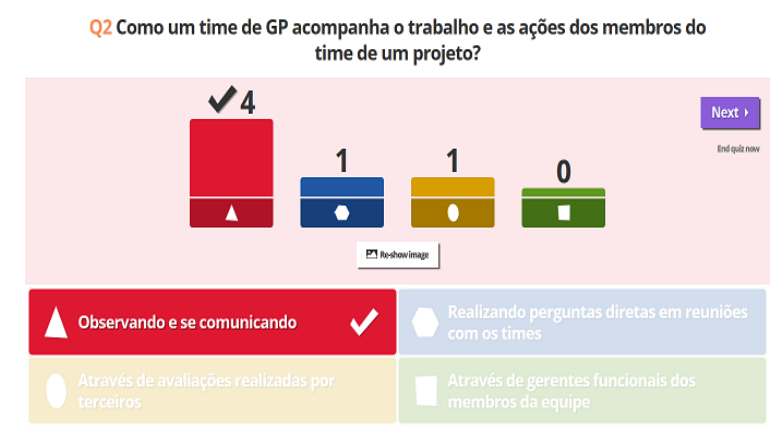

Figura 2. Quantidade de respostas por alternativa

Assim que o quiz game acabar aparecerá na tela do professor a classificação de pontos dos alunos mostrando as pontuações mais altas, e uma tela mostrando os acertos do vencedor. Após o fim da sessão, o professor poderá também baixar uma planilha com a pontuação dos jogadores e o desempenho de cada questão, no formato de arquivo de extensão XLS. 
V Congresso Brasileiro de Informática na Educação (CBIE 2016)

Anais do XXVII Simpósio Brasileiro de Informática na Educação (SBIE 2016)

\subsection{Execução}

A execução do jogo Kahoot! PMQuiz aconteceu nos semestres 2015/01 e 2015/02 nos laboratórios de informática do Departamento de Informática e Estatística (INE), da Universidade Federal de Santa Catarina (UFSC), com os alunos da disciplina INE5617Gerência de Projetos do curso de Sistemas de Informação e com os alunos da disciplina INE5427-Planejamento e Gestão de Projetos do curso de Ciência da Computação. No total, 71 alunos participaram da avaliação. A pesquisa realizada foi aprovada pelo Comitê de Ética sob parecer número 1.021.539 de 13/04/2015.

\subsection{Avaliação}

A avaliação foi realizada com objetivo de analisar o jogo em termos de motivação dos alunos, experiência de usuário e aprendizagem. Para isso, foi utilizado o modelo de avaliação de jogo educacionais MEEGA [Savi et al., 2011], que avalia a reação dos alunos após jogarem o jogo educacional. O modelo MEEGA oferece um questionário padronizado e validado para a coleta de dados após a aplicação de um jogo.

Análise dos dados. $O$ formato de resposta é uma escala Likert de 5 pontos, considerando a amplitude dos valores $(-2,-1,0,1,2)$, sendo que, o valor " -2 " representa total discordância e o valor "2" representa total concordância com a questão.

Em termos de motivação, os resultados podem ser visualizados na Figura 3. A avaliação mostrou que todos os itens da escala de motivação foram avaliados satisfatoriamente. Em especial, os itens referentes a relevância e atenção. Por outro lado, os itens referentes à satisfação do aluno, mesmo sendo avaliados positivamente, foram os itens que apresentaram maior número de indiferença e discordância. Isto pode ser justificado pois nem sempre os alunos podem perceber a necessidade da aplicação prática do conteúdo aprendido no jogo. Em geral, os resultados da escala de motivação mostram que o jogo educacional proporcionou um satisfatório grau de motivação nos alunos.

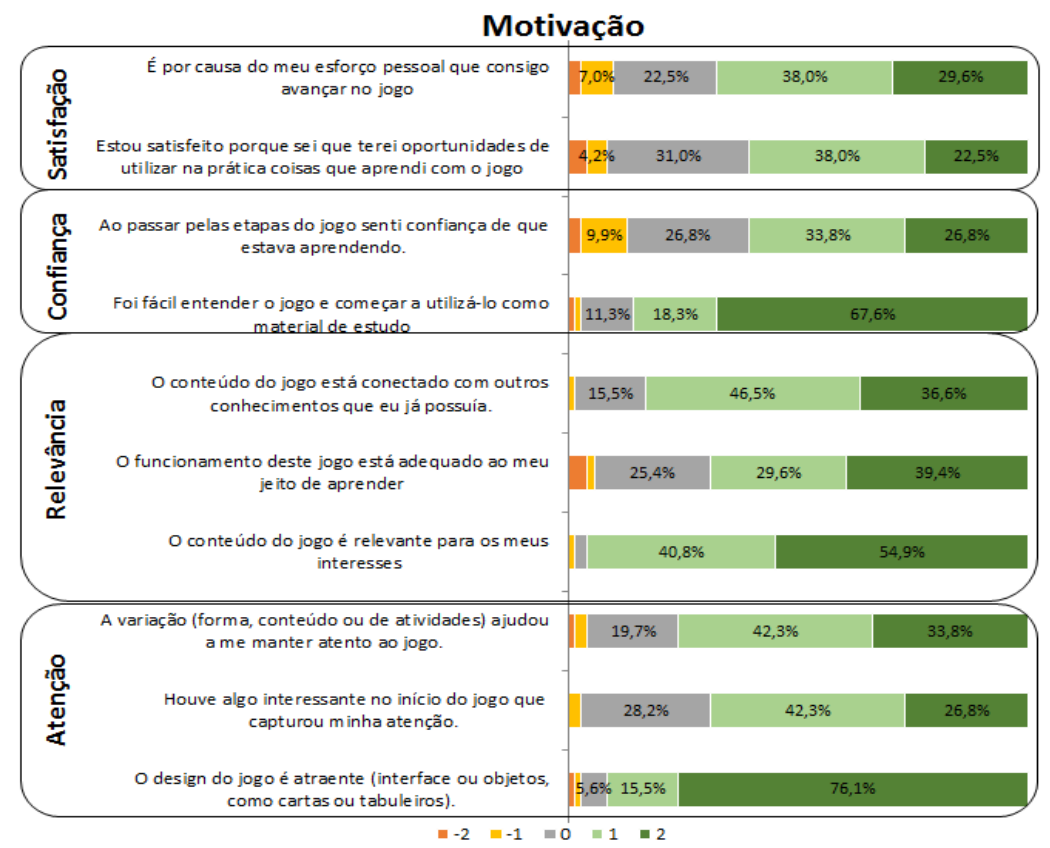

Figura 3. Avaliação da Motivação. 
V Congresso Brasileiro de Informática na Educação (CBIE 2016)

Anais do XXVII Simpósio Brasileiro de Informática na Educação (SBIE 2016)

Em termos de experiência do usuário, também é possível identificar que o jogo foi positivamente avaliado. A diversão, competição e desafio proporcionados pelo jogo foram os itens melhor avaliados. Isto se justifica pelo fato de o jogo fornecer um ranking dos alunos e apresentá-lo para toda a turma ao final de cada pergunta. Isto estimula os alunos a quererem acertar a próxima pergunta. Além disso, ao final de cada pergunta a resposta correta é discutida e justificada pelo professor, o que contribui para a revisão do conceito abordado na pergunta. Os resultados obtidos são visualizados na Figura 4.

\section{Experiência do Usuário}

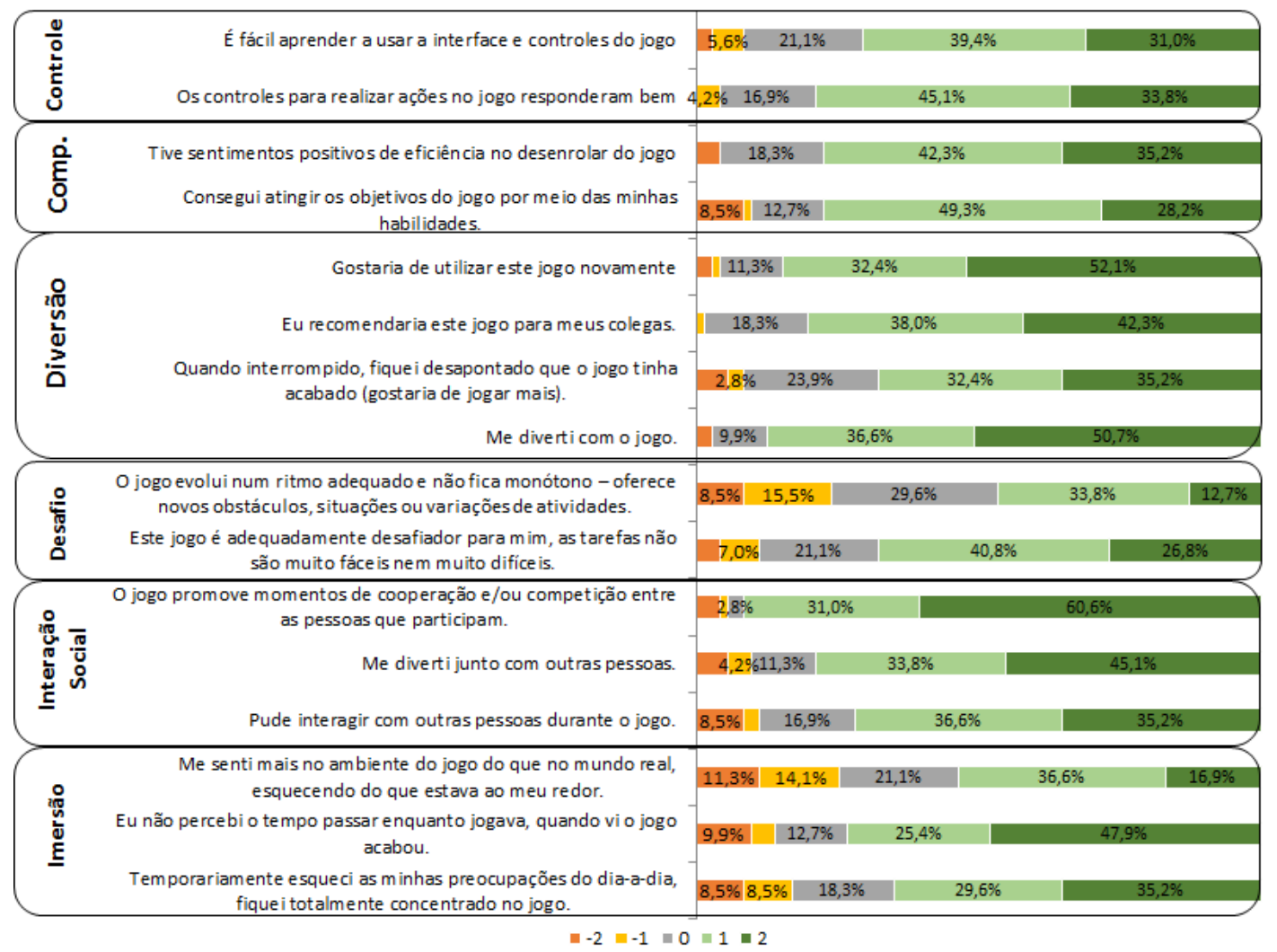

Figura 4. Avaliação da Experiência de Usuário.

Por outro lado, os itens com maior frequência de discordância e indiferença referem-se ao ritmo do jogo e também a imersão do jogador. Este resultado já era esperado, pois o gênero do jogo (quiz game) possui estas características. Como o jogo não possui uma narrativa e as interfaces são específicas para o gênero do jogo, a imersão pode não ocorrer. Em síntese, os dados sobre a experiência do usuário ao utilizar o jogo mostram que o Kahoot! PMQuiz proporcionou aos alunos uma boa experiência de usuário.

A avaliação da aprendizagem, apresentada na Figura 5, também mostrou resultados satisfatórios. Os alunos, em sua maioria, avaliaram que o jogo contribuiu para a sua aprendizagem na disciplina e também que o jogo foi eficiente para a aprendizagem, quando comparado com outras atividades realizadas na disciplina. Outro fator analisado no questionário foi o nível de conhecimento alcançado por meio do jogo, conforme apresenta a Figura 6. Na autoavaliação do nível de conhecimento, os alunos foram convidados a quantificar em uma nota de 0 a 5 o quanto consideravam relembrar, 
V Congresso Brasileiro de Informática na Educação (CBIE 2016)

Anais do XXVII Simpósio Brasileiro de Informática na Educação (SBIE 2016)

compreender e aplicar suas competências sobre a área de conhecimento de escopo, antes e após participarem da aplicação do jogo. De acordo com os resultados da autoavaliação, observou-se um acréscimo em todos os níveis de conhecimento dos alunos.

Aprendizagem

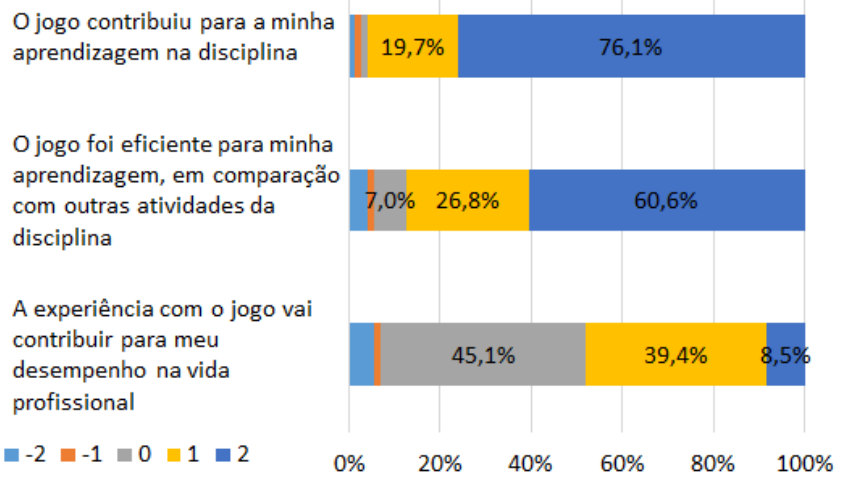

Figura 5. Avaliação da Aprendizagem.
Nível de conhecimento

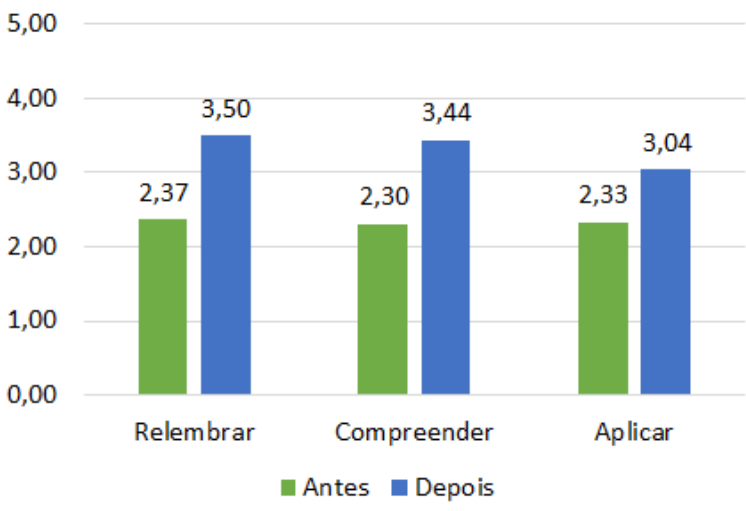

Figura 6. Autoavaliação do nível de conhecimento.

\section{Discussão}

O resultado das avaliações do jogo, aplicado nas duas disciplinas de gerenciamento de projetos de dois cursos de graduação, mostrou que o jogo Kahoot! PMQuiz proporcionou uma contribuição positiva para a aprendizado de gerenciamento de projetos.

Em geral, a avaliação do jogo foi positiva, mostrando que o Kahoot! PMQuiz motivou os alunos e também proporcionou uma boa experiência de usuário. O jogo foi considerado atrativo, com design agradável, conectado aos interesses e conhecimentos prévios dos alunos. Além disso, os alunos também demonstraram um alto grau de diversão, competição e interação social proporcionado pelo jogo.

\subsection{Ameaças à validade}

Para tratar as limitações na medição dos fatores de qualidade avaliados (conceitos subjetivos como motivação e experiência do usuário), foi usado o modelo MEEGA [Savi et al., 2011] um modelo de avaliação de jogos sistematicamente desenvolvido e amplamente validado. Embora a pesquisa tenha envolvido um número substancial de alunos, a aplicação da pesquisa nos próximos anos e em mais disciplinas pode ser uma alternativa para consolidar os dados coletados a partir deste trabalho. Além disso, como não houve um pré-teste medindo, por exemplo, o nível de conhecimento dos alunos antes da aplicação do jogo, pois causaria uma interrupção no fluxo das aulas, não foi possível identificar com exatidão o quanto cada aluno aprendeu sobre o assunto. No entanto, embora sem consenso, existem evidências de que a autoavaliação fornece informações confiáveis, válidas e úteis [Sitzmann et al., 2010]. 
V Congresso Brasileiro de Informática na Educação (CBIE 2016)

Anais do XXVII Simpósio Brasileiro de Informática na Educação (SBIE 2016)

\section{Conclusão e Trabalhos Futuros}

Este trabalho apresenta um quiz game educacional digital para a revisão de conhecimentos de gerenciamento de projetos em cursos superiores da área de computação. O Kahoot foi utilizado como plataforma para o desenvolvimento do quiz game, que foi desenvolvido seguindo o processo de desenvolvimento de jogos educacionais ENgAGED. Com base no ENgAGED foi possível customizar a ferramenta Kahoot e criar o quiz game Kahoot! PMQuiz levando em conta as características necessárias para que o jogo fosse efetivo educacionalmente. O Kahoot! PMQuiz foi aplicado e avaliado em duas disciplinas de gerenciamento de projetos e obteve um feedback positivo em termos de motivação, experiência do usuário e aprendizagem. A partir dessa avaliação, foi possível confirmar que o jogo educacional cumpriu com o seu objetivo de aprendizagem de proporcionar a revisão de conhecimentos sobre o gerenciamento de projetos em disciplinas da graduação de forma divertida e motivadora.

Para trabalhos futuros uma extensão da ferramenta pode ser implementada, englobando as funcionalidades relatadas em feedbacks pelos alunos, como a possibilidade de uso da ferramenta em modo off-line e classificação dos alunos disponível durante todo o jogo.

\section{Agradecimentos}

Este trabalho foi apoiado pelo $\mathrm{CNPq}$ (Conselho Nacional de Desenvolvimento Científico e Tecnológico), uma entidade do governo brasileiro focada no desenvolvimento científico e tecnológico e pela CAPES (Coordenação de Aperfeiçoamento de Pessoal de Nível Superior).

\section{Referências}

ABES. (2015). Associação Brasileira das Empresas de Software (Brasil) (Org.). Mercado Brasileiro de Software - Panorama e Tendências / Edição 2015 - Dados de 2014. Disponível em: < http://www.abessoftware.com.br/dados-do-setor/dados-2014>. Acesso em: 14 dez. 2015.

ACM/AIS/IEEE-CS The Joint Task Force for Computing Curricula. (2005). Computing Curricula 2005 - The Overview Report.

Backlund, P. and Hendrix, M. (2013). Educational games - Are they worth the effort? A literature survey of the effectiveness of serious games. In: 5th Int. Conf. on Games and Virtual Worlds for Serious Applications. Poole, GB.

Battistella, P. E. and Gresse von Wangenheim, C. (2015a). ENgAGED: Processo de Desenvolvimento de Jogos para Ensino em Computação. Relatório Técnico INCoD/GQS.01.2015.P, Instituto Nacional de Convergência Digital, Universidade Federal de Santa Catarina. Florianópolis, Brasil.

Battistella P. E. and Gresse von Wangenheim, C. (2016). Caracterização do Público-Alvo de Jogos Educacionais na área de Computação. In: Workshop sobre Educação em Computação, (pp. 20162025). Porto Alegre, RS.

Battistella, P. E., Wangenheim, A., and Gresse von Wangenheim, C. (2012). SORTIA - Um Jogo para Ensino de Algoritmo de Ordenação: Estudo de caso na Disciplina de Estrutura de Dados. In: XXIII Simpósio Brasileiro de Informática na Educação, (pp.1-10). Rio de Janeiro, RJ. 
V Congresso Brasileiro de Informática na Educação (CBIE 2016)

Anais do XXVII Simpósio Brasileiro de Informática na Educação (SBIE 2016)

Cassettari, F. T. Estudo de Caso: Uso de um Quiz Game para Revisão de Conhecimentos em Gerenciamento de Projetos. 2015. Trabalho de Conclusão de Curso. Graduação em Sistemas de Informação - Universidade Federal de Santa Catarina.

Connolly, T. M., Boyle, E. A., MacArthur, E., Hainey, T., \& Boyle, J. M. (2012). A systematic literature review of empirical evidence on computer games and serious games. Computers \& Education, 59(2), 661-686.

Dempsey, J., Rasmussen, K., and Lucassen, B. (1996). The instructional gaming literature: Implications and 99 sources. Technical Report 96-1. College of Education, University of South Alabama, AL.

Ferreira, B. M., Rivero, L., Lopes, A., Marques, A. B., and Conte, T. (2014). UsabiliCity: Um Jogo de Apoio ao Ensino de Propriedades de Usabilidade de Software Através de Analogias. In: XXV Simpósio Brasileiro de Informática na Educação, (pp. 1273-1282). Dourados, MS.

Germano, A. S. M., Aroca, S. C., Lopes, C. E. F., and Cunha, D. M. (2011). Integração do quiz como ferramenta de aprendizagem numa disciplina de astronomia na modalidade a distância. In Simpósio Nacional de Educação em Astronomia, (pp.1-7). Rio de Janeiro, RJ.

Gresse von Wangenheim, C., Carvalho, O. P., and Battistella, P. E. (2013). Ensinar a Gerência de Equipes em Disciplinas de Gerência de Projetos de Software. In: Revista Brasileira de Informática na Educação, 21(1), pp.15-22.

Kitchenham, B. (2010). Systematic literature reviews in software engineering - A tertiary study. Information and Software Technology, 52(1), 792-805.

Medeiros, R. A., Moreira, I. A. T., Barros, N. M., Leite, C. R. M., Lima, R. W., and Marques, L. P. S. (2013). GameES: Um Jogo para a Aprendizagem de Engenharia de Software. In: XXIV Simpósio Brasileiro de Informática na Educação, (pp. 945-949). Campinas, SP.

Natvig, L. and Line, S. (2004). Age of Computers - game-based teaching of computer fundamentals. In: 9th Annual Conf. on Innovation and Technology in Computer Science Education. Leeds, UK.

Parsons, P. (2011). Preparing computer science graduates for the 21st Century. Teaching Innovation Projects, 1(1), article 8.

PMI - Project Management Institute. Disponível em: <https://brasil.pmi.org/>. Acesso em: 14 maio 2016.

Savi, R., Gresse von Wangenheim. C., and Borgatto, A. (2011). Um Modelo de Avaliação de Jogos Educacionais na Engenharia de Software. In: XXV Simpósio Brasileiro de Engenharia de Software, (pp.194-203). São Paulo, SP.

Silva, M. A. A. and Dantas, A. (2014). KLouro: Um jogo educacional para motivar alunos iniciantes em programação. In: XXV Simpósio Brasileiro de Informática na Educação, (pp. 702-711). Dourados, MS.

Silva, T. R., Medeiros, T. J., and Aranha, E. H. S. (2014). Jogos Digitais para Ensino e Aprendizagem de Programação: uma Revisão Sistemática da Literatura. In: XXV Simpósio Brasileiro de Informática na Educação, (pp. 692-701). Dourados, MS.

Sitzmann, T., Ely, K., Brown, K. G., and Bauer, K. N. (2010). Self-Assessment of Knowledge: A Cognitive Learning or Affective Measure? Academy of Management Learning \& Education, 9(2), 169-191.

Standish Group. (2015). CHAOS Report 2015.

Wang, I. W., Ofsdahl T., and Sorstein O. K. M. (2008). An Evaluation of a Mobile Game Concept for Lectures. In: 21st Conf. on Software Engineering Education and Training. Charleston, USA. 